\title{
Structure of water adsorbed on a single graphene sheet
}

\author{
M. C. Gordillo* \\ Departamento de Sistemas Físicos, Químicos y Naturales, Facultad de Ciencias Experimentales, Universidad Pablo de Olavide, \\ Carretera de Utrera, $\mathrm{km} \mathrm{1,} 41013$ Sevilla, Spain \\ J. Martî́ \\ Departament de Física i Enginyeria Nuclear, Universitat Politècnica de Catalunya, B4-B5 Campus Nord, 08034 Barcelona, \\ Catalonia, Spain
}

(Received 2 June 2008; revised manuscript received 23 July 2008; published 22 August 2008)

\begin{abstract}
We present the result of molecular-dynamics simulations of water adsorbed on top of a single graphene layer at temperatures between 25 and $50{ }^{\circ} \mathrm{C}$. The analysis of the energy per particle and the density profiles indicate that the behavior of the adsorbed liquid is similar to the case of multiple graphene layers (graphite) with the only difference being the values of configurational energy. Other structural properties, such as stability ranges, hydrogen bond distributions, and molecular orientations are also presented.
\end{abstract}

DOI: 10.1103/PhysRevB.78.075432

PACS number(s): 68.08.De, 61.30.Hn, 61.20.Qg, 31.15.xv

\section{INTRODUCTION}

In recent years, the family of carbon forms has increased considerably with the discovery of fullerenes, carbon nanotubes, and other related structures. This has somehow made decay on the interest on the well-known graphite, which is basically a stack of flat layers separating each other in the $z$ direction by $3.35 \AA$. In these sheets, carbon atoms are located in the nodes of a honeycomb lattice and are joined by covalent bonds while the interlayer interaction is of dispersive type. However, it was recently reported that it was possible to isolate one of this layers, creating a different form of carbon termed graphene. ${ }^{1,2}$ As in the case of carbon nanotubes, its discovery started a rush to study all of its properties, particularly the electronic ones. ${ }^{3-6}$

As in the case of carbon nanotubes, the graphene properties as an adsorbent species could be of great interest. One can check, for instance, the different behavior, if any, of any adsorbate in the presence of a variable number of graphene sheets from single graphene to graphite. This would allow us to tune the influence of the substrate attraction on the properties of the adsorbed species when the surface itself is kept the same. Thus, our goal in the present paper will be to study the adsorption of water on top of a single graphene sheet and compare it to results when more graphene layers are added. This is a relatively different subject because, even though there are many studies related to what happens to water inside slit pores $^{7-9}$ and, in particular, near graphite surfaces, ${ }^{10-17}$ there were relatively few attempts to know what happens to water on top of a single hydrophobic surface $^{18,19}$ and none related to what happens on this purely two-dimensional (2D) adsorbent. There are, however, simulations about what happens to water inside slit pores limited by single graphene sheets. ${ }^{20,21}$ In this paper, we explore the possible influence of the substrate on the energetics and structure of the adsorbed species, studying other properties such as the orientations of the water molecules in the two interfaces: graphene water and water vacuum.

\section{METHOD AND SIMULATION DETAILS}

We considered liquid water on top of one of several graphite sheets at temperatures between 298 and $323 \mathrm{~K}$, and densities ranging from $6.8 \times 10^{-2}$ to $0.85 \AA^{-2}$. Our simulation box was, in all cases, $34.4 \times 34.1 \times 170 \AA^{3}$ in the $x, y$, and $z$ directions, respectively. This means that, to vary the density, we had to change the number of water molecules. We started from a fully equilibrated high-density configuration and simply subtracted a given number of water molecules to reach the selected density, allowing the system to thermally equilibrate in subsequent simulation runs. No periodic boundary conditions were considered in the $z$ axis. Several series of simulations were made: one with only one graphene layer, located at $z=0$, and other with up to four sheets. When additional sheets were considered, they were located parallel to the first one, under it (at negative $z$ positions), and separated from each other by $3.35 \AA$. All carbon atoms were explicitly taken into account in the calculation with each carbon layer being displaced in the $(x, y)$ plane with respect to their neighbors to recreate the real structure of graphite. All the graphene sheets were considered to be rigid, i.e., the carbon atoms were not allowed to move in the simulation runs. All of this means that we aimed to describe the interactions of water with a model sheet or sheets of graphene. The deviations due to any kind of real defects or vibrations of the structure are out of the scope of this work, which was meant to be a contact on the subject.

Water-water and water-carbon interactions have been modeled by means of the flexible simple point charge (SPC) potential already used in previous works, ${ }^{22}$ being basically a combination of Coulomb interactions (water water) with Lennard-Jones ones (water water and water graphene). The Lennard-Jones parameters for the water-carbon interaction were $\sigma_{O C}=3.28 \AA, \sigma_{H C}=2.81 \AA, \epsilon_{O C}=46.79 \mathrm{~K}$, and $\epsilon_{H C}$ $=15.52 \mathrm{~K}$. The long-ranged electrostatic interactions have been calculated by means of three-dimensional (3D) Ewald sums since, as it was demonstrated by Spohr, ${ }^{23}$ and by Yeh and Berkowitz, ${ }^{24}$ such procedure is fully equivalent to the $2 \mathrm{D}$ Ewald procedure, which corresponds to systems with periodic boundary conditions in only two dimensions. The only requirement that we need to impose is a box length in the perpendicular direction to graphene, namely the $z$ axis in our case, of at least five times the size of the water-graphene set along $z$ direction. No explicit cutoff was considered for the 
carbon-water potential, meaning that all the carbon-water interactions within the simulation cell indicated above were taken into account. The cutoffs in the calculation of waterwater interactions have been fixed at half of the box lengths.

A leap-frog Verlet integration algorithm with coupling to a thermal bath has also been employed, ${ }^{25}$ i.e., we moved within the parameters corresponding to the so-called molecular-dynamics ensemble, i.e., number of molecules, volume and total energy are fixed and the total linear momentum of the system is kept to zero, allowing temperature to suffer small fluctuations around a fixed average value. Our integration time step was $0.5 \mathrm{fs}$. Translational and internal degrees of freedom were equilibrated separately. The equilibration in all cases ran for at least $50 \mathrm{ps}$ and the averages were calculated in runs of lengths longer than 125 ps.

One of our goals is to characterize the range of densities in which the system is thermodynamically stable. To do so, it is not enough to compute the configurational energy of the system but there is the need to calculate the Helmholtz free energy. However, since the free energy cannot be directly obtained from a molecular-dynamics simulation, we have estimated it in the same way as in previous works for similar systems. ${ }^{20}$ The procedure starts from proposing a functional form for the free energy as a function of surface density and temperature, $F(\rho, T)$ :

$$
F(\rho, T)=\sum_{i=0}^{3} \sum_{j=1}^{3} b_{i j} \rho^{i} T^{1-j}+\text { ideal poliatomic gas terms }
$$

Given that

$$
E=-T^{2} \frac{\partial(F / T)}{\partial T}
$$

if we propose a form for $F(\rho, T)$, we can obtain the corresponding one for $E(\rho, T)$ simply by standard derivation scheme. We have then a polynomial whose coefficients are the $b_{i j}^{\prime} s$ in Eq. (1). If we fit the simulation data to that polynomial, we obtain the corresponding coefficients that we can substitute back in Eq. (1) to obtain the Helmholtz free energy. From the properties of this function, we can deduce that the system is stable at any particular pair of $\rho$ and $T$. In all cases, the quality of the fits is good, having the $\chi^{2}$ values for degree of freedom of around one.

\section{RESULTS}

\section{A. Thermodynamic stability}

In Fig. 1 we show the results we used to perform the least-square fittings that would allow us to obtain the free energy of the system. From top to bottom we have the total energy corresponding to temperatures of 323, 310, and 298 $\mathrm{K}$ versus water density. This density was obtained as the number of water molecules divided by the surface area of the simulation cell, i.e., it is a surface density. In all cases we observe that, after a relatively steep decrease in the energy, the trend forms something similar to a plateau for higher densities. The limiting value for this plateau would be the

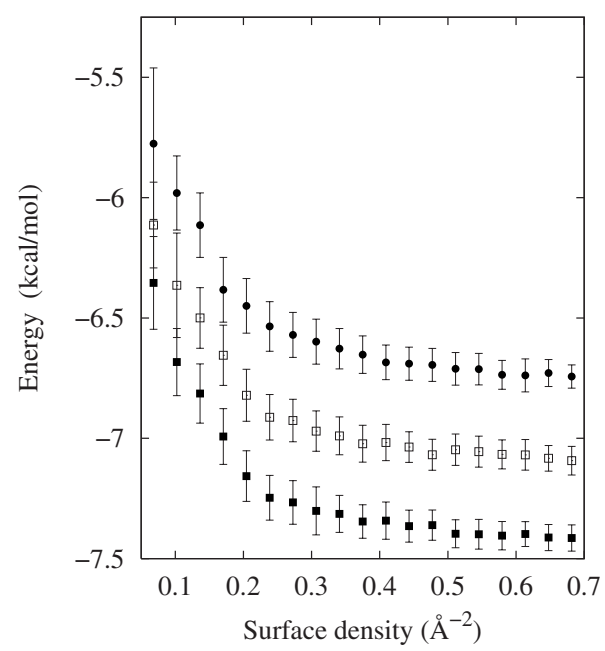

FIG. 1. Energy per molecule for water on top of a single graphene sheet: 50 (circles), 37 (open squares), and $25^{\circ} \mathrm{C}$ (full squares). The densities correspond to the number of water molecules divided by the surface of the simulation cell.

corresponding bulk water at the temperature at which the simulations are done. In any case, no minimum in the energy nor any inflection point was observed, at least not in the temperature range considered.

The influence of the substrate attraction in the adsorption energy could be seen with the help of Fig. 2. There, we displayed energy versus water density at $298 \mathrm{~K}$ for one, two, and three graphene layers. We observe the same trend as in the previous figure, i.e., the convergence to a plateau at large enough densities, but with energy limits noticeably different for two and three layers compared to those of a single graphene sheet. It can be also seen that the energy curves for more than two carbon sheets are similar to each other within the error bars. This is obviously the case for a greater number of layers as well, meaning that the energy limit for water at

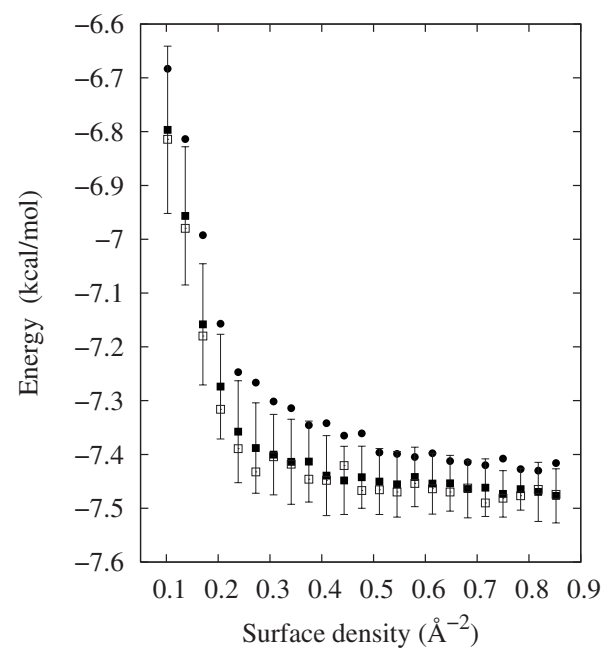

FIG. 2. Same as in Fig. 1 but only for water at 298 K. Energy per molecule for a single graphene sheet (full circles); energies for two graphene layers (full squares); energies for three graphene planes (open squares). Error bars are only displayed for the second case, being similar in the other two. 


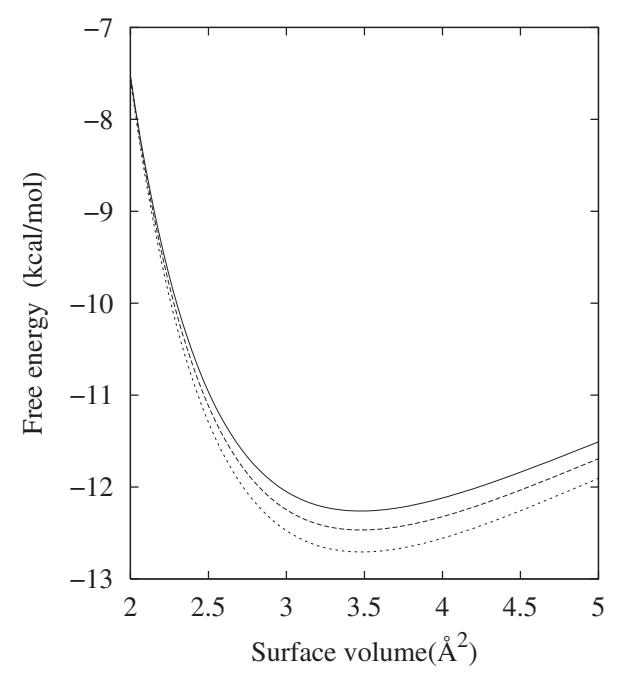

FIG. 3. Free energy per molecule obtained from the fit already discussed in the text. 298 (full line), 310 (long-dashed line), and $323 \mathrm{~K}$ (dashed line). $x$ axis indicates the inverse of the surface density displayed in previous figures.

room temperature adsorbed on real graphite is reached only with two graphene layers. This means that the properties as adsorbent of bilayer graphene will be similar to those of graphite, at least in the temperature range considered.

With the help of Fig. 3 we can establish the stability range of water on top of a single graphene sheet. There, we represent the free energy obtained from a fit to the energy values displayed in Fig. 1. The method was outlined in the previous section. From top to bottom are free energies for 298, 310, and $323 \mathrm{~K}$. One feature is immediately apparent: there is a single minimum for all temperatures considered, meaning that only arrangements with inverse surface densities greater than approximately $3.5 \AA^{2}$ are stable, i.e., those with a positive pressure. In our case, this means that at least 340 molecules are adsorbed per simulation cell.

\section{B. Density profiles and hydrogen bonding}

Figure 4 gives us an idea about the structure of adsorbed water at room temperature, corresponding to the full line to the minimum stable density. In all cases, a thin interfacial layer of $5 \AA$ wide is formed, as is well known for water near carbon-based structures. ${ }^{17,20,21}$ In particular, the structure of water adsorbed in paraffinlike plates ${ }^{26}$ is very similar to the one reported in Fig. 4, which suggests that the clue factor in determining water structure is the hydrophobic nature of the surface independently of the particular arrangement of surface atoms. In our stable systems, one can see that two well definite layers of water are formed, and after that, an increase in the number of molecules only produces the formation of a slab similar in density to that of bulk water. Obviously, that slab ends in a water-vacuum interface about $5 \AA$ wide in the $z$ direction.

The lowest density profile is similar to the one stable at low densities when water is confined between two parallel graphene layers separated by $31 \AA . .^{20,21}$ The influence of the number of graphene layers and temperature in this profile

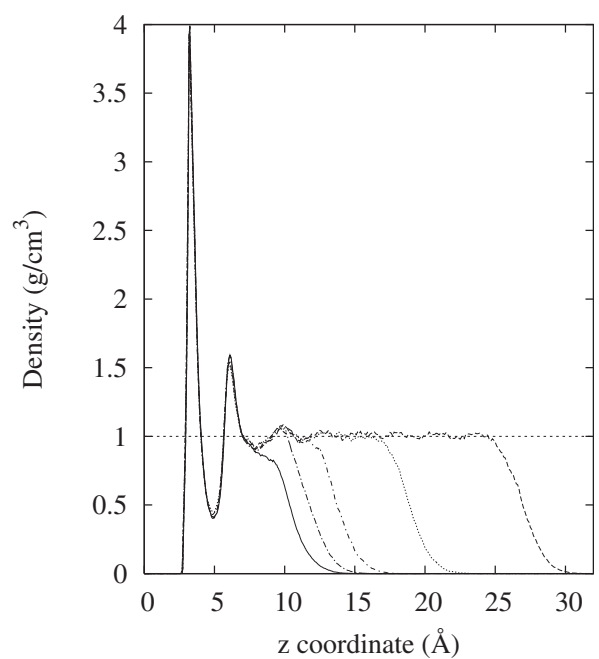

FIG. 4. Oxygen density profiles for several water surface densities on top of graphene. When more molecules are added, the tail of the density distribution moves to higher distances of the graphene layer. Densities from left (full line) to right (dashed line): 0.29, $0.34,0.41,0.58$, and 0.85 molecules $/ \AA^{2}$. After a zone influenced by the substrate, we found that the density is equal to one of the bulk (dotted horizontal line).

can be seen in Fig. 5. The data for a density of 0.51 molecule $\AA^{2}$ is similar in all the cases studied. The bottom part of the figure shows the effects of a double graphene layer while the upper part is the density profile for a single graphene sheet at $323 \mathrm{~K}$ compared to $298 \mathrm{~K}$. One sees easily that the profiles are practically identical in all cases, i.e., neither the temperature nor the number of carbon layers significantly affect the adsorbate, within the temperature range analyzed. Nevertheless, if other ranges were considered (supercooled or high temperature regimes, for instance), we could not establish how density profiles would be from the data reported in the present work and, consequently, further analysis would be necessary.

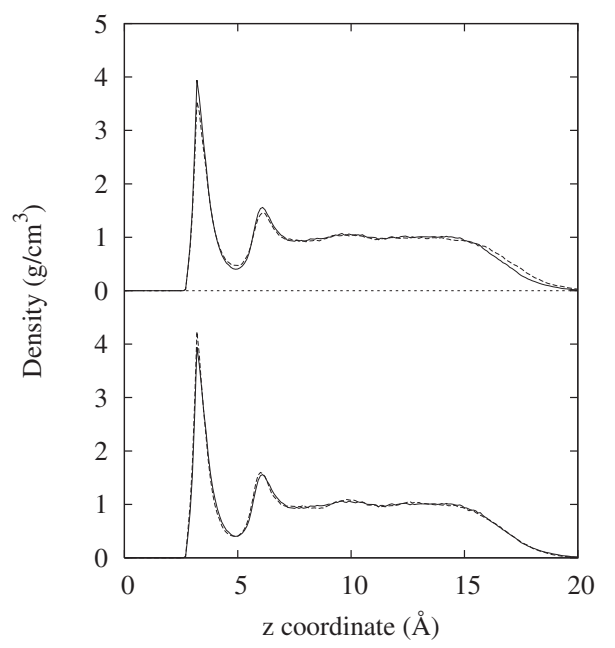

FIG. 5. Oxygen density profiles of water. Top figure: Single graphene layer with a water density of 0.51 molecules / $\AA^{2}(298 \mathrm{~K}$, full line; $323 \mathrm{~K}$, dashed line). Bottom figure: Temperature of $298 \mathrm{~K}$ and water density of 0.51 molecules $/ \AA^{2}$ (single graphene layer, full line; two graphene layers, dashed line). 


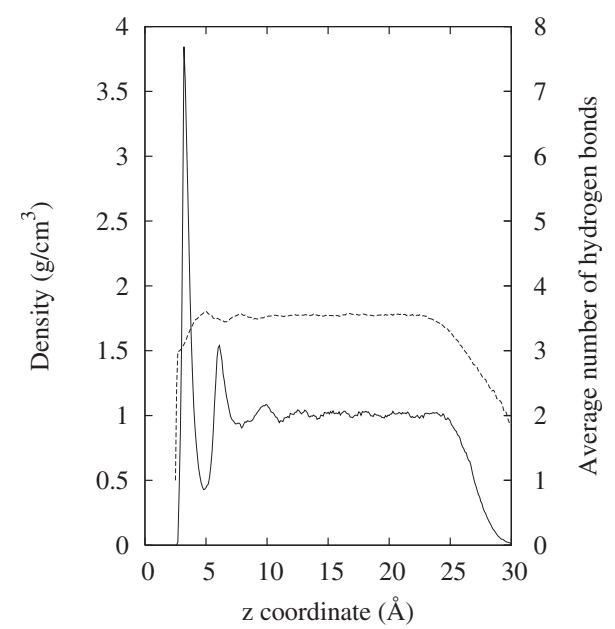

FIG. 6. Oxygen density profile for a water density of 0.85 molecules $/ \AA^{2}$ (full line), the largest studied. Dashed line indicates the average number of hydrogen bonds for a water molecule in the corresponding $z$ coordinate.

The relationship between the density profile and the average number of hydrogen bonds $\left\langle n_{H B}\right\rangle$ can be deduced from Fig. 6. There, we represent data for a density of 0.85 molecule $\AA^{2}$, fairly representative of what happens for other densities. We observe that $\left\langle n_{H B}\right\rangle \sim 3$ for water molecules closer to the surface, then $\left\langle n_{H B}\right\rangle$ quickly increases to a bulklike value of 3.5 , and decreases again in the watervacuum interface. This is similar to what happens in other hydrophobic surfaces due to the existence of dangling bonds, consisting of hydrogen atoms pointing directly to the graphene surface. 7,12,17,20,27,28 It is well known that the molecules having such bonds are unable to form more than three hydrogen bonds since one of its $\mathrm{OH}$ molecular bonds is not available.

However, this would maximize the number of bonds formed. On the other hand, water, immediately on top of this first layer of three bonds, is perfectly able to form as many hydrogen bonds as it can with molecules located on top or below it. That is the reason why the number of hydrogen bonds increases after the first peak in the density profile. This conclusion is supported by the analysis of the water orientations, both for the $\mathrm{OH}$ direction and for the water dipole moment direction with respect to the carbon layer, as it will be discussed in the following section.

\section{Water orientations}

An analysis of orientations of the molecular $\mathrm{OH}$ direction as measured with respect to the unit vector perpendicular to the graphene surface, i.e., using the tilt angle $\theta$ as defined in other works, ${ }^{20,29}$ confirms the existence of those dangling OH's (maxima at $\sim 1$ in Fig. 7) for molecules located at distances to the surface of $5 \AA$ or less (up to the first minimum in the density profiles). The orientation of the second molecular $\mathrm{OH}$ bond can be deduced, taking into account the molecular water angle $\left(\sim 104^{\circ}\right)$. Thermal disorder accounts in part of other orientations, even though the dipole distribution for the same type of molecules given in Fig. 8 suggests

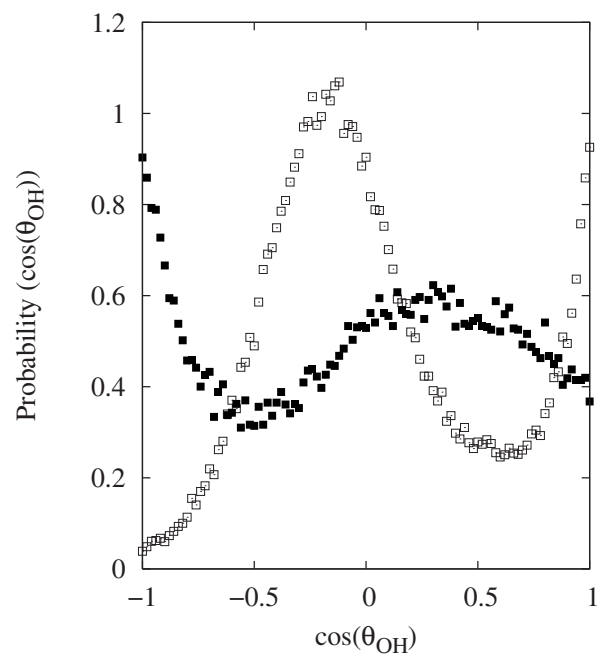

FIG. 7. Distribution of probabilities of $\cos \left(\theta_{\mathrm{OH}}\right)$ (see text) in arbitrary units for a density of 0.58 molecules $/ \AA^{2}$. Water closest to the single graphene sheet, up to $5 \AA$ of distance (open squares), and water in the outer $2 \AA$ wide slab (full squares). In the zone, in between the former two, the distribution is nearly flat and it is not shown for simplicity.

another contribution from molecules located in planes roughly parallel to the surface (maxima around $0^{\circ}$ ), which would merge within the single peak in the $\mathrm{OH}$ distribution. These results are in good agreement with recent findings. ${ }^{17,20,21,27}$ Configurations with a dangling bond would, in return, contribute in broadening considerably the maximum in the dipole distributions (from $\sim 0.61$ down).

From data reported in both Figs. 7 and 8, we can deduce the orientation of the water molecules located from the end of the bulk region and up. The molecules in the intermediate zone between the water-graphene and water-vacuum interfaces show no preferential orientation, i.e., they have a bulklike profile and are not reported in the plots. The $\mathrm{OH}$ angle distribution indicates that water in the outer layer orientates with a bond pointing outward from the bulk intermediate

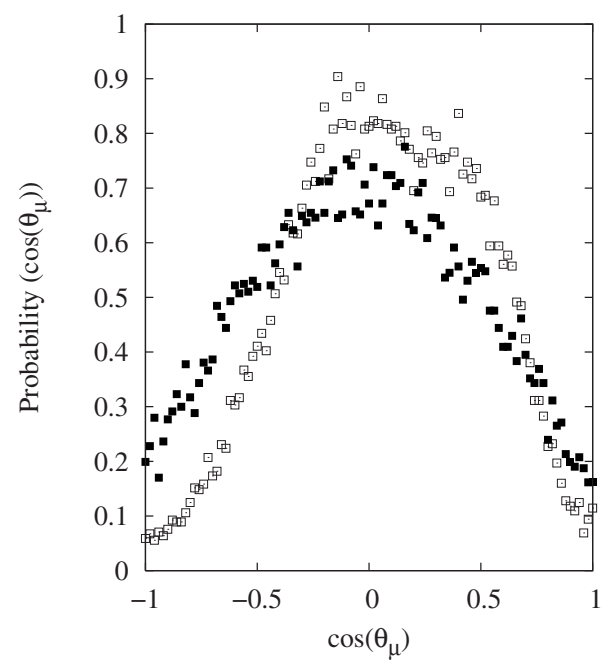

FIG. 8. Same as in Fig. 7 but for the projection of the water dipole moment direction (see text). 
region. This correlates well with the maximum around 0.20 that will correspond to the second $\mathrm{OH}$ bond of the molecule. As already discussed, the nonzero value in between those maxima is probably due to thermal disorder. This means that, close to a surface, water tends to maximize the number of hydrogen bonds by pointing one bond outside the bulk, creating a distribution that is basically the mirror image of that of the layer closest to the graphene. The differences can be accounted for from the fact that vacuum exerts no attraction on the water molecules, smoothing somehow the probability functions. This averaging can also be seen in the dipole distribution of Fig. 8, which can be interpreted in the same lines already discussed for water in the first layer, and taking in consideration its mirrorlike characteristics.

\section{CONCLUDING REMARKS}

We studied the structural characteristics of water adsorbed on top of a single graphene layer by means of moleculardynamics simulations built on a flexible SPC force field for water-water interactions, and Lennard-Jones forces between water and carbon atoms. In our simulations, polarizability of water has not been explicitly taken into account although trivial changes of the molecular dipole moment of water are considered because ours is a flexible potential allowing variations of internal water geometry. This is a relevant aspect to be considered since, as has been recently reported, ${ }^{30}$ the influence of water polarizability on the air/water interface when ionic species (iodide) are present into the system. We do believe that, in the case of ionic solutions, the role of polarizability should be taken into account in deeper detail than that employed here by us. Pursuing this objective, investigation on this issue is currently being undertaken in our laboratory.

We found that, as an adsorbent, graphene is quite similar to graphite with the only sizeable difference being the value of the binding energy of water to the substrate. However, this energy is not distinguishable to that of graphite (multiple graphene layers) once we have added a second graphene layer to the first one. The structure on top of graphene is quite similar to that of one observed for water in between relatively wide graphene pores, as it was expected. The only significant difference is the existence of a water-vacuum interface that presents characteristics similar to that of the first layer adsorbed but with its features smoothed out, particularly when water orientations are considered.

\section{ACKNOWLEDGMENTS}

M.C.G thanks the Spanish Ministry of Education and Science (MEC) and the Junta de Andalucia for financial support under Grants No. FIS2006-02356 and No. P06-FQM-01869, respectively. J.M. gratefully acknowledges financial support from the Direcció General de Recerca de la Generalitat de Catalunya (Grant No. 2005SGR-00779) and the MEC (Grant No. FIS2006-12436-C02-01). *cgorbar@upo.es

${ }^{\dagger}$ Corresponding author. jordi.marti@upc.edu

${ }^{1}$ K. S. Novoselov, A. K. Geim, S. V. Morozov, D. Jiang, Y. Zhang, S. V. Dubonos, I. V. Grigorieva, and A. A. Firsov, Science 306, 666 (2004).

${ }^{2}$ K. S. Novoselov, D. Jiang, F. Schedin, T. J. Booth, V. V. Khotkevich, S. V. Morozov, and A. K. Geim, Proc. Natl. Acad. Sci. U.S.A. 102, 10451 (2005).

${ }^{3}$ K. S. Novoselov, A. K. Geim, S. V. Morozov, D. Jiang, M. I. Katsnelson, I. V. Grigorieva, S. V. Dubonos, and A. A. Firsov, Nature (London) 438, 197 (2005).

${ }^{4}$ Y. Zhang, Y. Tan, H. L. Stormer, and P. Kim, Nature (London) 438, 201 (2005).

${ }^{5}$ C. Berger, Z. Song, T. Li, X. Li, A. Y. Ogbazghi, R. Feng, Z. Dai, A. N. Marchenkov, E. H. Conrad, P. N. First, and W. A. Heer, J. Phys. Chem. B 108, 19912 (2004).

${ }^{6}$ A. K. Geim and K. S. Novoselov, Nat. Mater. 6, 183 (2007).

${ }^{7}$ C. Y. Lee, J. A. McCammon, and P. J. Rossky, J. Chem. Phys. 80, 4448 (1984).

${ }^{8}$ S. B. Zhu and G. W. Robinson, J. Chem. Phys. 94, 1403 (1991).

${ }^{9}$ A. Striolo, A. Chialvo, P. T. Cummings, and K. E. Gubbins, Langmuir 19, 8583 (2003).

${ }^{10}$ G. Nagy, J. Electroanal. Chem. 409, 19 (1996).

${ }^{11}$ P. Cabrera Sanfelix, S. Holloway, K. W. Kolasinski, and G. R. Darling, Surf. Sci. 532-535, 166 (2003).

${ }^{12}$ A. Pertsin and M. Grunze, J. Phys. Chem. B 108, 1357 (2004).

${ }^{13}$ R. Zangi, J. Phys.: Condens. Matter 16, S5371 (2004).
${ }^{14}$ M. Schoen and D. J. Diestler, J. Chem. Phys. 109, 5596 (1998).

${ }^{15}$ K. Lum, D. Chandler, and J. D. Weeks, J. Phys. Chem. B 103, 4570 (1999).

${ }^{16}$ T. M. Truskett, P. G. Debenedetti, and S. Torquato, J. Chem. Phys. 114, 2401 (2001).

${ }^{17}$ G. Cicero, J. K. Grossman, E. Schwegler, F. Gygi, and G. Galli, J. Am. Chem. Soc. 130, 1871 (2008).

${ }^{18}$ M. C. Gordillo and J. Martí, J. Chem. Phys. 117, 3425 (2002).

${ }^{19}$ J. Martí and M. C. Gordillo, J. Chem. Phys. 119, 12540 (2003).

${ }^{20}$ J. Martí, G. Nagy, M. C. Gordillo, and E. Guàrdia, J. Chem. Phys. 124, 094703 (2006).

${ }^{21}$ G. Nagy, M. C. Gordillo, E. Guàrdia, and J. Martí, J. Phys. Chem. B 111, 12524 (2007).

${ }^{22}$ M. C. Gordillo and J. Martí, Chem. Phys. Lett. 329, 341 (2000).

${ }^{23}$ E. Spohr, J. Chem. Phys. 107, 6342 (1997).

${ }^{24}$ I.-C. Yeh and M. L. Berkowitz, J. Chem. Phys. 111, 3155 (1999).

${ }^{25}$ H. J. C. Berendsen, J. P. M. Postma, W. F. van Gunsteren, A. DiNola, and J. R. Haak, J. Chem. Phys. 81, 3684 (1984).

${ }^{26}$ N. Choudhury, J. Phys. Chem. B 112, 6296 (2008).

${ }^{27}$ N. Choudhury and B. M. Pettit, J. Am. Chem. Soc. 127, 3556 (2005).

${ }^{28}$ S. H. Lee and P. J. Rossky, J. Chem. Phys. 100, 3334 (1994).

${ }^{29}$ D. K. Hore, D. S. Walker, and G. L. Richmond, J. Am. Chem. Soc. 130, 1800 (2008).

${ }^{30}$ P. Jungwirth and D. J. Tobias, Chem. Rev. (Washington, D.C.) 106, 1259 (2006). 